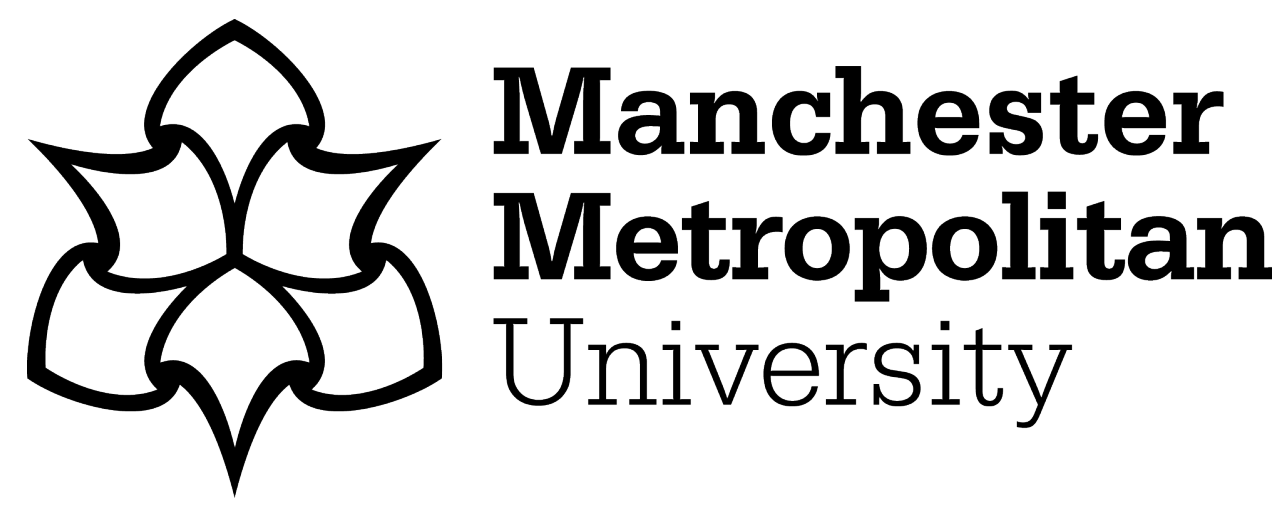

Thomas, Christopher, Dos'Santos, Thomas, Comfort, Paul and Jones, Paul A (2020) Male and female soccer players exhibit different knee joint mechanics during pre-planned change of direction. Sports Biomechanics. pp. 1-14. ISSN 1476-3141

Downloaded from: https://e-space.mmu.ac.uk/626766/

Version: Accepted Version

Publisher: Informa UK Limited

DOI: https://doi.org/10.1080/14763141.2020.1830160

Please cite the published version 


\section{Male and female soccer players exhibit different knee joint mechanics 2 during pre-planned change of direction}

3 Christopher Thomas ${ }^{\mathrm{a} *}$, Thomas Dos'Santos ${ }^{\mathrm{a}}$, Paul Comfort ${ }^{\mathrm{a}}$ and Paul A. 4 Jones $^{\mathrm{a}}$

$5{ }^{a}$ Directorate of Sport, Exercise and Physiotherapy. University of Salford, Salford, 6 Greater Manchester, UK

$7 \quad$ Address author correspondence to Christopher Thomas at c.thomas2@edu.salford.ac.uk

8 Christopher Thomas is a PhD fellow in Biomechanics and Strength and Conditioning at the 9 University of Salford, UK.

10 Thomas Dos'Santos is a PhD student in Biomechanics and Strength and Conditioning at the 11 University of Salford, UK.

12 Paul Comfort is a Reader in Strength and Conditioning and Program Leader of the MSc Strength 13 and Conditioning at the University of Salford, UK

14 Paul A. Jones is a Lecturer in Biomechanics and Strength and Conditioning at the University of 15 Salford, UK. 


\title{
Male and female soccer players exhibit different knee joint mechanics during pre-planned change of direction
}

\begin{abstract}
Change of direction manoeuvres are important in soccer and associated with noncontact anterior cruciate ligament injury, yet it is not known how the mechanics differentiate between males and females during $180^{\circ}$ turns. Twenty-eight soccer players (14 male and 14 female) performed $180^{\circ}$ turns with ground reaction forces collected over penultimate and final contacts. A two-way (contact $\mathrm{x}$ limb) multivariate analysis of variance (MANOVA) were run to examine differences between contact (penultimate and final) or limb (dominant and nondominant) for sagittal plane hip, knee and ankle peak angles and moments, and frontal plane knee abduction moments and angles between sexes. Average horizontal GRF was increased on the dominant limb, compared to nondominant and for the final contact compared to the penultimate contact. Knee abduction angles were increased in females compared to males, while the opposite was true for knee abduction moments. Statistically significant differences were evident, with increases in peak vertical GRF, peak hip flexion angle, peak knee flexion angle, peak knee extensor moment, and peak ankle dorsiflexion angle observed in the penultimate contact compared to final contact. The results indicate the penultimate contact during turns helps reduce loading on the final contact, yet male and female soccer players exhibit different knee joint mechanics during pre-planned change of direction.
\end{abstract}

Keywords: word; agility; anterior cruciate ligament; turns; injury; knee abduction moments, $180^{\circ}$ turns

\section{Introduction}

It has previously been observed that female players have higher rates of non-contact anterior cruciate ligament (ACL) injuries compared with males (Myklebust, Maehlum, Holm, \& Bahr, 1998). There is also evidence that knee joint mechanics are differentiated by sex during change of direction (CoD) tasks (cutting and turning), contributing to increased risk of ACL injury (Brophy, Silvers, Gonzales, \& Mandelbaum, 2010; McLean, Huang, \& van den Bogert, 2008; Sigward, Pollard, \& Havens, 2012). Several studies have shown that females display increased knee abduction angles (Malinzak, Colby, 
Kirkendall, Yu, \& Garrett, 2001; McLean, Lipfert, \& van den Bogert, 2004), knee abduction moments (McLean, Huang, \& van den Bogert, 2005; McLean et al., 2004; Sigward, Cesar, \& Havens, 2015; Sigward et al., 2012), vertical ground reaction forces (GRFs) (Yu, Lin, \& Garrett, 2006) and smaller knee flexion angles (Malinzak et al., 2001; McLean et al., 2004; Yu et al., 2006) as compared with their male counterparts during cutting and turning. Moreover, video analysis studies have revealed postures at initial contact such as a dorsiflexed ankle (Boden, Torg, Knowles, \& Hewett, 2009), abducted hip (Olsen, Myklebust, Engebretsen, \& Bahr, 2004), extended knee joint (Boden et al., 2009; Krosshaug et al., 2007; Olsen et al., 2004), and laterally flexed and rotated torso (Stuelcken, Mellifont, Gorman, \& Sayers, 2016) to be associated with ACL injuries during CoD. Similarly, laboratory studies have found these lower limb postures to increase knee abduction moments (Dempsey, Lloyd, Elliott, Steele, \& Munro, 2009; Jones, Herrington, \& Graham-Smith, 2016a, 2016b), which could lead to increased ACL strain (McLean, Su, \& van den Bogert, 2003) and subsequent injury (Hewett et al., 2005). Previous studies have investigated the influence of sex on CoD biomechanics (Fedie, Carlstedt, Willson, \& Kernozek, 2010; McLean et al., 2005, 2004; Sigward et al., 2015, 2012). Pollard et al. (2018) demonstrated healthy male and female participants exhibit similar lower extremity biomechanics during a $45^{\circ}$ side-step. These contrasting findings may be due to inconsistency in how the dominant limb is defined, or the velocity and magnitude of the CoD. For example, sex differences in knee abduction moments during $110^{\circ}$ turns have been observed, with females greater than males, but no differences were observed in $45^{\circ}$ cuts (Sigward et al., 2015). Similarly, females were found to exhibit increased knee valgus angles and internal knee adductor moments during 45 and $110^{\circ}$ cutting when compared with males (Sigward et al., 2012). Sharper CoD (i.e. $180^{\circ}$ turns) increase the relative lower body loading compared to shallow $\operatorname{CoD}\left(<60^{\circ}\right)$ and thus, 
require substantial braking over several footfalls prior to push-off. Previous work found

74 lower knee flexion angles, yet higher knee abduction angles during a $180^{\circ}$ turn compared to a $45^{\circ}$ cut (Cortes, Onate, \& Van Lunen, 2011), while reductions in knee flexion angle have been observed with sharper CoDs (Schreurs, Benjaminse, \& Lemmink, 2017). Furthermore, the knee joint has been found to play a primary role during the deceleration phase of shaper CoDs (Havens \& Sigward, 2015a). Further studies on the kinetics and kinematics during turning between male and female soccer players are required to fully understand the biomechanical requirements of $180^{\circ} \mathrm{CoD}$ and help optimise $\mathrm{CoD}$ performance and minimise knee joint loading.

It has been previously observed that up to $70 \%$ of non-contact ACL injuries occur during a cutting or CoD maneuver (Boden, Feagin, \& Garrett, 2000; Boden, Sheehan, Torg, \& Hewett, 2010). Previous research (Brophy et al., 2010), suggests limb dominance (kicking vs. support limb) to influence knee joint mechanics and ACL injury, specifically in soccer players. Although non-contact ACL injuries were evenly distributed (kicking $\operatorname{limb}=30 ;$ support limb $=28), 74 \%(20 / 27)$ of males suffered a non-contact ACL injury on the kicking limb, compared with $32 \%$ (10/31) of females. There have been several studies in the literature reporting the influence of limb dominance on $\mathrm{CoD}$ biomechanics

90 (Bencke et al., 2013; Brown, Wang, Dickin, \& Weiss, 2014; Greska, Cortes, Ringleb, 91 Onate, \& Van Lunen, 2016; Marshall et al., 2014; Mok, Bahr, \& Krosshaug, 2018; Pollard et al., 2018), and can be defined as the preferential use of one side of the body when performing a motor task, typically resulting in a more skilful and therefore dominant side

94 (Maloney, 2019). For example, the preferred limb to kick a ball in soccer or change direction is typically used to indicate limb dominance, and as such, could provide coaches and researchers information whether a limb is at heightened risk of increased loading, and

97 thus potential for injury, or not. Early work shows no differences in knee joint mechanics 
98 (knee flexion angle, knee abduction angle, knee internal rotation angle, and knee

99 abduction moment) during weight acceptance between preferred and non-preferred limbs

100 in female soccer players (Brown et al., 2014). In contrast, 20 collegiate female soccer

101 players were found to exhibit similar CoD biomechanics (hip and knee moments, and

102 GRFs) between dominant kicking) and nondominant (support) limbs (Greska et al.,

103 2016). Moreover, the dominant limb displayed increased peak knee flexion angles,

104 increased peak internal knee abduction moments, and increased peak vertical GRFs,

105 while the nondominant limb exhibited increased knee abduction angles at initial contact

106 and peak value and increased vertical GRF at peak knee abduction moment. Recently,

107 Thomas et al., (2017) reported that female soccer players adopt different braking

108 strategies between dominant and nondominant limbs in $180^{\circ}$ turns, whereby increased

109 horizontal braking force is placed on the penultimate contact by the nondominant limb

110 when turning off the dominant limb. Conversely, an increased force is placed on the final

111 contact when turning off the nondominant limb.

112 Improving our understanding of limb dominance during CoD may provide further insight

113 into the potential mechanisms of increased loading and help drive performance and injury

114 prevention programmes. Therefore, the primary aim of this study was to investigate

115 differences in kinematics (lower-limb joint angles) and kinetics (GRFs and moments) in

116 the sagittal and frontal planes, between males and females during $180^{\circ}$ turns. The

117 secondary aim was to investigate differences in braking strategy (penultimate vs. final

118 contact) on the dominant vs. nondominant limbs during $180^{\circ}$ turns in male and female

119 soccer players. Finally, this study aimed to explore kinematic and kinetic differences

120 between penultimate and final contact of $180^{\circ}$ turns. It was hypothesised that female

121 players would exhibit increased knee abduction angles and knee abduction moments

122 compared to males (Sigward et al., 2015, 2012). Furthermore, it was hypothesised that 
123 female soccer players would demonstrate increased horizontal GRF during the final 124 contact when turning off the nondominant limb (Thomas et al., 2017). It was hypothesised

125 that the penultimate contact would demonstrate increased knee joint flexion angles, peak

126 horizontal GRF, but lower average horizontal GRF during compared to the final contact

127 (Jones et al., 2016b)

\section{Methods}

\section{Participants}

130 This study included 28 male $(n=14$; age $=24.5 \pm 4.2$ years; height $=1.79 \pm 0.05 \mathrm{~m}$; body

131 mass $=78.5 \pm 9.6 \mathrm{~kg})$ and female $(n=14$; age $=20.6 \pm 0.6$ years; height $=1.65 \pm 0.07 \mathrm{~m}$;

132 body mass $=56.2 \pm 6.6 \mathrm{~kg}$ ) soccer players. All participants were of semi-professional

133 level and did not suffer from an ACL injury in the past, or any other lower-limb injury

134 within the last 6 months. Each player was in the preseason phase of training during his or

135 her participation in this study. All participants read and signed a written informed consent

136 form before participation, with consent from the parent or guardian of all participants

137 under the age of 18. Approval for the study was provided by the University of Salford's

138 Institutional Ethics Committee.

\section{Experimental Protocol}

140 Lower-limb kinetic and kinematic data were collected during $180^{\circ}$ turns (505 CoD test),

141 performed as fast as possible, on an indoor track (Mondo, SportsFlex, $10 \mathrm{~mm}$; Mondo

142 America Inc., Mondo, Summit, NJ, USA). The 505 involved running towards two force

143 platforms, whereby the first force platform was used to measure GRFs from the

144 penultimate foot contact (2nd to last foot contact with the ground during a pivot before

145 moving into a new intended direction), whilst the 2 nd force platform was used to measure 
146 GRFs from the final contact (last foot contact with the ground during a pivot before

147 moving into a new intended direction). Players were instructed to sprint to a line marked

148 on the central portion of 2 nd force platform, $15 \mathrm{~m}$ from the start, planting their left or

149 right foot on the line, turn $180^{\circ}$ and sprint back $5 \mathrm{~m}$ through the finish. Prior to maximal

150 trials participants performed at least 3 submaximal trials, turning off each limb at $75 \%$ of

151 perceived maximum effort. Players performed a minimum of six acceptable trials (3 left

152 and 3 right) in a randomised and counterbalanced order. If participants slid, turned

153 prematurely, or missed the force platform, the trial was discarded and subsequently

154 performed after a 2-minute rest.

155 Before the turn task, reflective markers (14 $\mathrm{mm}$ spheres) were placed on the

156 following bony landmarks: right and left iliac crests; anterior superior iliac spine;

157 posterior superior iliac spine; greater trochanter; medial epicondyle; lateral epicondyle;

158 lateral malleoli; medial malleoli; heel; and fifth, second, and first metatarsal heads using

159 double-sided adhesive tape. Each player wore a four-marker 'cluster set' (four

160 retroreflective markers attached to a lightweight rigid plastic shell) on the right and left

161 thigh and shin which approximated the motion of these segments during the dynamic

162 trials. All participants wore lycra shorts and female participants wore a compression top

163 (Champion Vapor, Champion, Winston-Salem, NC, USA). Standardised footwear

164 (Balance W490, New Balance, Boston, MA, USA) was provided for all participants to

165 control for shoe-surface interface.

\section{Data Analysis}

167 3D motions of these markers were collected during the turn trials using 10 Qualisys Oqus

1687 (Gothenburg, Sweden) infrared cameras $(240 \mathrm{~Hz})$ operating through Qualisys Track

169 Manager software (Qualisys, version 2.16, build 3520, Gothenburg, Sweden). The GRFs

170 were collected from two $600 \mathrm{~mm}$ x $900 \mathrm{~mm}$ AMTI (Advanced Mechanical Technology, 
171 Inc, Watertown, MA, USA) force platforms (Model number: 600900) embedded into the

172 running track, sampling at $1200 \mathrm{~Hz}$. From a standing trial, a lower extremity and trunk 6

173 degrees of freedom kinematic model was created for each player, including pelvis, thigh,

174 shank and foot using Visual3D software (C-motion, version 6.01.12, Germantown, USA).

175 This kinematic model was used to quantify the motion at the hip, knee and ankle joints

176 using a Cardan angle sequence $\mathrm{x}-\mathrm{y}-\mathrm{z}$ (Grood \& Suntay, 1983). The local coordinate

177 system was defined at the proximal joint centre for each segment. The static trial position

178 was designated as the subject's neutral (anatomical zero) alignment, and subsequent

179 kinematic measures were related to this position. Segmental inertial characteristics were

180 estimated for each participant (Dempster, 1955). The model used a CODA pelvis

181 orientation (Charnwood Dynamics Ltd., Leicestershire, UK) (Bell, Brand, \& Pedersen,

182 1989) to define the location of the hip joint centre. The knee and ankle joint centres were

183 defined as the mid-point of the line between lateral and medial markers. Lower limb joint

184 moments were calculated using an inverse dynamics approach (Winter, 2009) through

185 Visual3D software and were defined as external moments.

186 The trials were time normalised for each subject, for ground contact time of the

187 turn task. Initial contact was defined as the point after ground contact that the vertical

188 GRF was higher than $20 \mathrm{~N}$ and end of contact was defined as the point where the vertical

189 GRF subsided past $20 \mathrm{~N}$ for both penultimate and final contact. The weight acceptance

190 phase of ground contact was defined as from the instant of instant contact (vertical GRF

$191>20 \mathrm{~N}$ ) to the point of maximum knee flexion during ground contact as used previously

192 (Havens \& Sigward, 2015b; Jones et al., 2016b; Jones, Thomas, Dos'Santos, McMahon,

193 \& Graham-Smith, 2017). Joint coordinate and force data were smoothed in Visual3D with

194 a Butterworth low pass digital filter with cut-off frequencies of 12 and $25 \mathrm{~Hz}$,

195 respectively. Cut-off frequencies were selected based on a priori residual analysis 
196 (Winter, 2009) and visual inspection of the motion data.

197 For comparisons between penultimate and final contact, peak and average vertical

198 (Fz) and horizontal (Fx) GRFs were determined along with peak hip, knee and ankle

199 dorsiflexion angles and peak hip, knee and ankle moments in the sagittal plane during the

200 weight acceptance phase, and analysed in Microsoft Excel (version 2016, Microsoft

201 Corp., Redmond, WA, USA). Furthermore, peak knee abduction angles and knee

202 abduction moments were calculated during the final contact. Joint moment data were

203 normalised to body mass $(\mathrm{Nm} / \mathrm{kg})$. To evaluate the deceleration strategy from penultimate

204 to final contact, a final contact/penultimate contact horizontal (Fx component) horizontal

205 GRF ratio was also calculated (Jones et al., 2016b). In line with recent research

206 (Dos'Santos, Comfort, \& Jones, 2020), data were analysed based on the average of trial 207 peaks.

\section{Statistical Analyses}

209 Data are presented as either mean $\pm S D$. Normality of data was assessed by Shapiro-

210 Wilk's statistic, while homogeneity of variances was examined using Levene's test. A

211 two-way (contact $\mathrm{x}$ limb) multivariate analysis of variance (MANOVA) was used to

212 determine if differences exist between foot contact (penultimate and final) or limb

213 (dominant and nondominant) and between sexes (male and female) when considering all

214 dependent variables in the sagittal plane. Separate $2 \times 2(\operatorname{limb} \times$ sex $)$ repeated-measures

215 ANOVA were run to examine differences in completion time, horizontal GRF ratio, knee

216 abduction angles and knee abduction moments. Where significant differences were

217 found, Bonferroni post hoc analyses were completed to detect differences between

218 groups. The dominant limb was defined as the limb with the fastest time to completion

219 during CoD. All statistical analyses were performed in the Jamovi Project for Windows

220 (Jamovi Project, 2019) and the criterion for statistical significance was set at $\mathrm{p} \leq 0.05$. 


\section{Results}

Table 1 shows repeated measures ANOVAs for force-time characteristics, while

Table 2 shows repeated measures ANOVAs for sagittal and frontal plane peak joint angle and moment data.

The average approach speed for males $(5.2 \pm 0.3 \mathrm{~m} / \mathrm{s})$ was significantly faster $(\mathrm{p}$ $<0.001)$ than females $(4.7 \pm 0.3 \mathrm{~m} / \mathrm{s})$. A significant difference $(\mathrm{p}<0.001)$ in completion time was observed between limbs whereby the dominant limb was faster than nondominant. Average horizontal GRFs were increased $(p=0.013)$ for dominant limb compared with nondominant.

A significant difference $(\mathrm{p}=0.034)$ in average horizontal GRF ratio was observed between sexes, with females demonstrating an increased ratio than males. There was no other statistically significant main effect or interaction for both average and peak horizontal GRF ratio.

For the variables peak hip flexion angle, peak vertical GRF, peak knee flexion angle, peak knee extensor moment and peak ankle dorsiflexion angle, although an interaction was not present, there were main effects for contact, indicating increased values in the penultimate compared to final contact when both sexes were combined. Yet, the opposite was true for peak plantarflexor moment whereby values were increased in the final contact compared to penultimate contact. There was no main effect or interaction present for the variable peak horizontal GRF.

A significant difference $(\mathrm{p}<0.001)$ in peak knee abduction angle was observed

244 between sexes, with females demonstrating increased angles than males. In contrast, peak 245 knee abduction moments were significantly $(\mathrm{p}=0.012)$ increased for males compared to 
246 females. Furthermore, significant differences $(p=0.006)$ were noted between males and

247 females in peak hip flexion angle, with males demonstrating increased values than

248 females. When considering completion time, males were significantly faster $(\mathrm{p}<0.001)$

249 compared to females.

\section{Discussion and Implications}

Although previous studies have considered the influence of the limb dominance on knee injury risk factors during pre-planned tasks (Brown, Donelon, Smith, \& Jones, 2016; Brown et al., 2014; Greska et al., 2016), this is the first study to evaluate the interaction of penultimate and final contact on such factors in both males and females. This is important given that turning movements are common in both sexes (Bloomfield,

256 Polman, \& O’Donoghue, 2007; Boden et al., 2010; Brophy et al., 2010). The aims of this study were to: (1) evaluate sex differences in lower-limb kinetics and kinematics between males and females during $180^{\circ}$ turns, (2) compare lower-limb kinetics and kinematics between dominant and nondominant limbs, and (3) explore kinetic and kinematic

260 differences between penultimate and final contacts of $180^{\circ}$ turns. The most striking result to emerge from the data is that females demonstrated increased knee abduction angles compared to males $(\mathrm{p}<0.001$ ), but the opposite was evident when analysing knee abduction moment $(\mathrm{p}=0.012)$. Consistent with previous research, these results suggest that $\mathrm{CoD}$ biomechanics are sex-specific and thus, should be interpreted accordingly when informing training and injury prevention interventions. Specifically, practitioners must acknowledge from a technique perspective the 'performance-injury conflict' when coaching and performing $\mathrm{CoD}$, ensuring players have optimal $\mathrm{CoD}$ mechanics and physical capacity to tolerate the associative knee joint loading.

Our primary finding was that knee abduction angles were increased in females compared to males, whereas knee abduction moments were increased in males compared 
to females. This is in agreement with previous work whereby increased knee abduction angles were observed in females compared to males (Sigward et al., 2015), yet in the same study, females exhibited increased knee abduction moments, which is in contrast to our findings. A possible explanation for this might be that Sigward et al. (2015) used a $110^{\circ}$ side-step whilst the current study used a $180^{\circ}$ turn. Recent work (Schreurs et al., 2017) indicates knee valgus moments tend to stabilise when changing direction to magnitudes $>90^{\circ}$ in both males and females. It could be that athletes subconsciously restrain this moment from becoming increased when changing direction to increased magnitudes $\left(90-180^{\circ}\right)$. Yet, the male players in the current study demonstrated significantly $(\mathrm{p}<0.001)$ faster time to completion than females, due to faster average approach speeds, thus likely contributing to great knee abduction moments. This is in agreement with previous research reporting increases in knee abduction moments from faster running velocities during $60^{\circ}$ (Kimura \& Sakurai, 2013) and $135^{\circ}$ (Nedergaard, Kersting, \& Lake, 2014) CoD. This finding has important implications for developing performance and injury prevention programmes. Specifically, faster and sharper CoD increase knee joint loading but are also required for successful performance to evade or

287 close an opponent; thus, causing a performance-injury conflict from a technique perspective (Dos'Santos, Thomas, Comfort, \& Jones, 2018), but can be mediated by an athlete's physical capacity. Further research might explore the influence physical capacities (strength and power measures) on lower-limb kinetics and kinematics, as this may help drive sex-specific CoD and ACL prevention programming.

This study has shown that average horizontal GRF was lower for the penultimate contact compared to the final contact. This finding is consistent with that of (Jones et al., 2016b) who found lower average horizontal GRF in the penultimate contact relative to the final contact. The same authors also found peak vertical GRF values to be higher in 
the penultimate contact compared to the final contact, which also agrees with the findings

297 of the current study. Also, ground contact time were shown to be longer in the final 298 contact of turns than the penultimate contact $(0.52 \pm 0.08 \mathrm{~s}$ vs. $0.38 \pm 0.07 \mathrm{~s})$, resulting in

299 an increased horizontal braking impulse (impulse $=$ force $\mathrm{x}$ [change in momentum]) in

300 the final contact (Jones et al., 2016b). Taken together, these findings indicate, during

301 turns, the need to bring the horizontal velocity to zero before turning and accelerating

302 back the other way, therefore more substantial braking takes place during the final

303 contact. This may present a problem when athletes may not have the physical capacities

304 (neuromuscular control, high levels of strength) to cope with the increased loading. Thus,

305 it is essential to develop holistic training programmes to optimally prepare and enhance

306 CoD performance and reduce risk of injury. Specifically, strength, plyometric, sprint,

$307 \mathrm{CoD}$ and combination training are all found to be effective modalities of improving CoD

308 ability (Falch, Rædergård, \& van den Tillaar, 2019), while others have found reductions

309 in KAM resulting from technique modification interventions (Dempsey et al., 2009;

310 Jones, Barber, \& Smith, 2015). Most recently, Dos'Santos (Dos'Santos, McBurnie,

311 Comfort, \& Jones, 2019) found improvements in CoD completion time and cutting

312 technique following a 6-week $\mathrm{CoD}$ technique intervention in male youth soccer players,

313 indicating CoD technique training, in addition to normal skills and strength training

314 improves cutting performance and movement quality. Indeed, athletes with increased

315 levels of isokinetic eccentric knee extensor strength are shown to be better able to

316 decelerate during the penultimate contact from faster approach velocities during $180^{\circ}$

317 turns (Jones et al., 2017). Furthermore, peak horizontal braking forces during penultimate

318 contact are shown to significantly associate with CoD performance times (Graham-Smith,

319 Atkinson, Barlow, \& Jones, 2009) and horizontal GRF ratio (Dos' Santos, Thomas, Jones,

320 \& Comfort, 2017), with faster athletes demonstrating significantly lower horizontal 
321 braking force ratio than slower athletes. These findings may help us to understand the 322 interaction between strength, speed, and technique regarding CoD performance and risk 323 of injury.

324 In this study, females demonstrated increased average horizontal GRF ratio 325 compared to males, indicating an increased proportion of braking took place during the 326 final contact relative to the penultimate contact, compared to males. This result is in 327 accord with recent studies indicating faster CoD performances to exhibit lower horizontal 328 GRF ratio as compared with slower performances (Dos' Santos et al., 2017). Also, earlier studies found lower horizontal GRF ratio to associate with lower knee abduction moments 330 during turns (Jones et al., 2016a), yet this was in female participants and turning off one 331 leg only. Further work is required to evaluate the role of the penultimate contact and final contact in $180^{\circ}$ turns in male and female soccer players to better understand the optimal technique for changing direction.

The joint angle data revealed a significant main effect for peak hip flexion angle, indicating increased hip flexion was observed during the penultimate contact compared to final contact. This finding is likely to be related to help absorb loading through an increased range of motion compared with final contact, thus facilitating longer braking force application, thus impulse, resulting in an increased reduction in whole-body velocity (impulse $=$ change in momentum). Indeed, the role of the penultimate contact has been described as a 'preparatory step' demonstrating hip and knee flexion throughout

341 the stance phase as the athlete transitions from penultimate contact to final contact (Jones

342 et al., 2016b). This helps provide an optimal body position at final contact (lower centre 343 of mass) and allows the final contact leg to be planted out in front of the body. Another 344 important finding was that males exhibited increased hip flexion angles during weight 345 acceptance than females. There are similarities between the finding in this study and those 
346 described by (Sigward et al., 2015) whereby males demonstrated increased hip abduction

347 angle at initial contact than females. It seems possible that these results are because male

348 athletes better prepare themselves for the CoD by either absorbing GRFs (hip flexion) or

349 pre-rotate to the new direction (hip abduction). This finding, while preliminary, suggests

350 that male athletes may better self-regulate their CoD technique, which may lead to a faster

351 overall CoD performance. Indeed, earlier work suggests a lack of hip flexion/extensor

352 moments to be a gender technique deficit, potentially leading to increased knee loading

353 (Pollard, Sigward, \& Powers, 2007).

$354 \quad$ For the variables peak knee flexion angle and peak knee extensor moment, there

355 were significant main effects for contact, with increased values observed during

356 penultimate contact compared to final contact. These results match those of earlier studies

357 whereby the penultimate contact resulted in increased knee flexion angles and knee

358 extensor moments in $180^{\circ}$ turns (Graham-Smith et al., 2009; Greig, 2009; Jones et al.,

$3592016 \mathrm{~b})$. These results are likely to be related to the fact that, during $180^{\circ}$ turns, the knee

360 goes through an increased range of knee flexion during penultimate contact compared to

361 final contact. These findings suggest that increased knee flexion is maintained in the

362 transition from penultimate contact to final contact to lower centre of mass and allow for

363 an optimal final contact, with data showing maximum knee flexion typically occurs at the

364 end of penultimate contact ground contact in $180^{\circ}$ turns (Jones et al., 2016b). Another

365 finding was that increased peak ankle dorsiflexion angle was observed in the penultimate

366 contact compared to final contact, but the opposite was true for peak plantarflexor

367 moment, with increased values in the final contact compared to penultimate contact.

368 These findings are in agreement with those who found increased ankle dorsiflexor

369 moments during final contact compared to penultimate contact (Jones et al., 2016b).

370 These results may be explained by the fact that participants initially made the final contact 
371

372

373

374

with a forefoot plant, evoking an ankle dorsiflexor moment, whereas during penultimate contact an initial rearfoot plant may have led to increased plantarflexor moments. Furthermore, increased ankle dorsiflexion occurs to help absorb the loading and facilitate longer braking force application during penultimate contact in $180^{\circ}$ turns.

Overall, the findings of this study provide insights into the role of limb dominance during the task of $180^{\circ}$ turns. While many studies have explored biomechanical differences between limbs during CoD (Brown et al., 2016; Mok et al., 2018; Pollard et al., 2018; Thomas et al., 2017), this investigation is the first to examine the differences between dominant and nondominant limb across penultimate contact and final contact, in male and female soccer players. The results of this study indicate a significant main effect for limb for the variable average horizontal GRF; whereby increased values were observed when turning off the dominant limb compared to the nondominant limb. A way of interpreting this might be that when turning with the dominant limb increased average braking forces are experienced across the final two foot contacts, likely due to technical and coordination differences. The other main effects observed in this study suggest average horizontal GRFs are increased in the final contact than penultimate contact, which agrees with past literature (Jones et al., 2016a, 2016b). This combination of findings may provide some support for the conceptual premise that the role of limb dominance on CoD biomechanics may be less in such shallow angles of direction change $\left(<90^{\circ}\right)$, but more so for sharper $\operatorname{CoD}\left(90-180^{\circ}\right)$. Thus, further research is required to investigate whether limb dominance influences the braking strategy during these manoeuvres.

A limitation of the current study is the pre-planned execution of the CoD task, whereas unanticipated $\mathrm{CoD}$ has been shown to elevate knee joint loads during cutting (Besier, Lloyd, Ackland, \& Cochrane, 2001). In addition, it can only be assumed that 
knee valgus and knee abduction moments are risk factors for ACL injury due to the lack

397 of evidence. Also, females wore compression garments, but males did not; therefore, it is

398 unknown the amount of movement artefact when comparing markers and clusters

399 attached to a compression garment compared with those attached to skin. Furthermore,

400 the findings of the current study can only be extrapolated to male and female soccer

401 participants performing $180^{\circ}$ turns. Except for knee abduction angles and moments, this

402 study only featured lower-limb joint angles and moments in the sagittal plane. Despite

403 hip abduction and rotation angles, such as the motion on the frontal and transverse planes,

404 are commonly investigated in cutting studies (Kristianslund, Faul, Bahr, Myklebust, \&

405 Krosshaug, 2014; Kristianslund \& Krosshaug, 2013), whole-body deceleration takes

406 place in the sagittal plane during $180^{\circ}$ turns. In future studies, it might be possible to

407 investigate the influence of these parameters on braking strategy and knee joint mechanics

408 during $180^{\circ}$ turns.

409 Acknowledgements

410 The authors thank the participants for taking part in the study.

\section{Disclosure Statement}

412 No potential conflict of interest was reported by the authors.

\section{References}

414 Bell, A. L., Brand, R. A., \& Pedersen, D. R. (1989). Prediction of hip joint centre location from external landmarks. Human Movement Science, 8(1), 3-16.

416 Bencke, J., Curtis, D., Krogshede, C., Jensen, L. K., Bandholm, T., \& Zebis, M. K.

417 (2013). Biomechanical evaluation of the side-cutting manoeuvre associated with 418 ACL injury in young female handball players. Knee Surgery, Sports

419 Traumatology, Arthroscopy, 21(8), 1876-1881. 
Besier, T. F., Lloyd, D. G., Ackland, T. R., \& Cochrane, J. L. (2001). Anticipatory effects on knee joint loading during running and cutting maneuvers. Medicine and Science in Sports and Exercise, 33, 1176-1181.

Bloomfield, J., Polman, R., \& O’Donoghue, P. (2007). Turning movements performed during FA Premier League soccer matches. Journal of Sports Science and Medicine, 6(10), 9.

Boden, B. P., Feagin Jr, J. A., \& Garrett Jr, W. E. (2000). Mechanisms of anterior cruciate ligament injury. Orthopedics, 23, 573.

Boden, B. P., Sheehan, F. T., Torg, J. S., \& Hewett, T. E. (2010). Non-contact ACL injuries: Mechanisms and risk factors. Journal of the American Academy of Orthopaedic Surgeons, 18, 520-527.

Boden, B. P., Torg, J. S., Knowles, S. B., \& Hewett, T. E. (2009). Video analysis of anterior cruciate ligament injury abnormalities in hip and ankle kinematics. American Journal of Sports Medicine, 37, 252-259.

Brophy, R., Silvers, H. J., Gonzales, T., \& Mandelbaum, B. R. (2010). Gender influences: The role of leg dominance in ACL injury among soccer players. British Journal of Sports Medicine, 44(10), 694-697.

Brown, N., Donelon, T., Smith, L. C., \& Jones, P. A. (2016). An investigation into the effect of limb preference on knee mechanics and braking strategy during a $180^{\circ}$ change of direction task: An exploratory study. Journal of Sports Sciences, 34(sup1), s57. https://doi.org/10.1080/02640414.2016.1260807

Brown, S., Wang, H., Dickin, D. C., \& Weiss, K. J. (2014). The relationship between leg preference and knee mechanics during sidestepping in collegiate female footballers. Sports Biomechanics, 13, 351-361. 
444 Cortes, N., Onate, J., \& Van Lunen, B. (2011). Pivot task increases knee frontal plane loading compared with sidestep and drop-jump. Journal of Sports Sciences, 29, 83-92.

Dempsey, A. R., Lloyd, D. G., Elliott, B. C., Steele, J. R., \& Munro, B. J. (2009). Changing sidestep cutting technique reduces knee valgus loading. American Journal of Sports Medicine, 37, 2194-2200.

Dempster, W. T. (1955). Space requirements of the seated operator: Geometrical, kinematic, and mechanical aspects of the body, with special reference to the limbs. Michigan State Univ East Lansing.

Dos' Santos, T., Thomas, C., Jones, P. A., \& Comfort, P. (2017). Mechanical determinants of faster change of direction speed performance in male athletes. Journal of Strength and Conditioning Research, 31(3), 696-705.

Dos'Santos, T., Comfort, P., \& Jones, P. A. (2020). Average of trial peaks versus peak of average profile: Impact on change of direction biomechanics. Sports Biomechanics, 19(4), 483-492.

Dos'Santos, T., McBurnie, A., Comfort, P., \& Jones, P. A. (2019). The effects of sixweeks change of direction speed and technique modification training on cutting

Dos'Santos, T., Thomas, C., Comfort, P., \& Jones, P. A. (2018). The effect of angle and velocity on change of direction biomechanics: An angle-velocity trade-off. Sports Medicine, 48(10), 2235-2253.

Falch, H. N., Rædergård, H. G., \& van den Tillaar, R. (2019). Effect of different physical training forms on change of direction ability: A systematic review and meta-analysis. Sports Medicine-Open, 5(1), 53. 
Fedie, R., Carlstedt, K., Willson, J. D., \& Kernozek, T. W. (2010). Effect of attending to a ball during a side-cut maneuver on lower extremity biomechanics in male and female athletes. Sports Biomechanics, 9, 165-177.

Graham-Smith, P., Atkinson, L., Barlow, R., \& Jones, P. (2009). Braking characteristics and load distribution in 180 degree turns. In Proceedings of the 5th Annual UKSCA Conference., 6-7.

Greig, M. (2009). The influence of soccer-specific activity on the kinematics of an agility sprint. European Journal of Sport Science, 9, 23-33. https://doi.org/10.1080/17461390802579129

Greska, E. K., Cortes, N., Ringleb, S. I., Onate, J. A., \& Van Lunen, B. L. (2016). Biomechanical differences related to leg dominance were not found during a

Grood, E. S., \& Suntay, W. J. (1983). A joint coordinate system for the clinical description of three-dimensional motions: Application to the knee. Journal of Biomechanical Engineering, 105, 136-144.

Havens, K. L., \& Sigward, S. M. (2015a). Joint and segmental mechanics differ between cutting maneuvers in skilled athletes. Gait and Posture, 41, 33-38. http://dx.doi.org/10.1016/j.gaitpost.2014.08.005

Havens, K. L., \& Sigward, S. M. (2015b). Whole body mechanics differ among running and cutting maneuvers in skilled athletes. Gait and Posture, 42, 240-245.

490 Hewett, T. E., Myer, G. D., Ford, K. R., Heidt, R. S., Colosimo, A. J., McLean, S. G., ... Succop, P. (2005). Biomechanical measures of neuromuscular control and valgus loading of the knee predict anterior cruciate ligament injury risk in 
female athletes a prospective study. American Journal of Sports Medicine, 33, 492-501.

495

496

497

498

499

500

501

502

503

504

505

506

507

508

509

510

511

512

513

514

515

516

517

Jamovi Project, jamovi. (2019). Jamovi (Version 0.9)[Computer Software].

Jones, P. A., Herrington, L. C., \& Graham-Smith, P. (2016a). Technique determinants of knee abduction moments during pivoting in female soccer players. Clinical Biomechanics, 31, 107-112.

Jones, P. A., Herrington, L., \& Graham-Smith, P. (2016b). Braking characteristics during cutting and pivoting in female soccer players. Journal of Electromyography and Kinesiology, 30, 46-54.

Jones, P. A., Thomas, C., Dos'Santos, T., McMahon, J. J., \& Graham-Smith, P. (2017). The role of eccentric strength in $180^{\circ}$ turns in female soccer players. Sports, $5(2), 42$.

Jones, P., Barber, O., \& Smith, L. (2015). D2. S2. 5 (3). Changing pivoting technique reduces knee valgus moments: Taken from Day 2. Free CommunicationsBiomechanics and Motor Behaviour. Journal of Sports Sciences, 33(1), S62.

Kimura, K., \& Sakurai, S. (2013). A sidestep cut preparation strategy decreases the external load applied to the knee joint. International Journal of Sport and Health Science, 11, 109-117.

Kristianslund, E., Faul, O., Bahr, R., Myklebust, G., \& Krosshaug, T. (2014). Sidestep cutting technique and knee abduction loading: Implications for ACL prevention exercises. British Journal of Sports Medicine, 48, 779-783. https://doi.org/10.1136/bjsports-2012-091370

Kristianslund, Eirik, \& Krosshaug, T. (2013). Comparison of drop jumps and sportspecific sidestep cutting: Implications for anterior cruciate ligament injury risk screening. American Journal of Sports Medicine, 41(3), 684-688. 
518 Krosshaug, T., Nakamae, A., Boden, B. P., Engebretsen, L., Smith, G., Slauterbeck, J.

519 R., ... Bahr, R. (2007). Mechanisms of Anterior Cruciate Ligament Injury in

520 Basketball: Video Analysis of 39 Cases. American Journal of Sports Medicine,

$521 \quad 35,359-367$. https://doi.org/10.1177/0363546506293899

522 Malinzak, R. A., Colby, S. M., Kirkendall, D. T., Yu, B., \& Garrett, W. E. (2001). A

523 comparison of knee joint motion patterns between men and women in selected

$524 \quad$ athletic tasks. Clinical Biomechanics, 16, 438-445.

525 Maloney, S. J. (2019). The relationship between asymmetry and athletic performance: A

526 critical review. The Journal of Strength \& Conditioning Research, 33(9), 2579-

$527 \quad 2593$.

528 Marshall, B. M., Franklyn-Miller, A. D., King, E. A., Moran, K. A., Strike, S. C., \&

529 Falvey, E. C. (2014). Biomechanical factors associated with time to complete a

$530 \quad$ change of direction cutting maneuver. Journal of Strength and Conditioning

$531 \quad$ Research.

532 McLean, S. G., Huang, X., \& van den Bogert, A. J. (2005). Association between lower

533 extremity posture at contact and peak knee valgus moment during sidestepping:

534 Implications for ACL injury. Clinical Biomechanics, 20, 863-870.

535 McLean, S. G., Huang, X., \& van den Bogert, A. J. (2008). Investigating isolated

536 neuromuscular control contributions to non-contact anterior cruciate ligament

537 injury risk via computer simulation methods. Clinical Biomechanics, 23, 926-

538 936. https://doi.org/10.1016/j.clinbiomech.2008.03.072

539 McLean, S. G., Lipfert, S. W., \& van den Bogert, A. J. (2004). Effect of gender and defensive opponent on the biomechanics of sidestep cutting. Medicine and Science in Sports and Exercise, 36, 1008-1016. 
McLean, S. G., Su, A., \& van den Bogert, A. J. (2003). Development and validation of a 3-D model to predict knee joint loading during dynamic movement. Journal of Biomechanical Engineering, 125, 864-874.

Mok, K.-M., Bahr, R., \& Krosshaug, T. (2018). Reliability of lower limb biomechanics in two sport-specific sidestep cutting tasks. Sports Biomechanics, 17(2), 157167.

Myklebust, G., Maehlum, S., Holm, I., \& Bahr, R. (1998). A prospective cohort study of anterior cruciate ligament injuries in elite Norwegian team handball. Scandinavian Journal of Medicine and Science in Sports, 8, 149-153.

Nedergaard, N. J., Kersting, U., \& Lake, M. (2014). Using accelerometry to quantify deceleration during a high-intensity soccer turning manoeuvre. Journal of Sports Sciences, 32, 1897-1905. https://doi.org/10.1080/02640414.2014.965190

Olsen, O.-E., Myklebust, G., Engebretsen, L., \& Bahr, R. (2004). Injury mechanisms for 555 anterior cruciate ligament injuries in team handball a systematic video analysis. The American Journal of Sports Medicine, 32, 1002-1012.

Pollard, C. D., Norcross, M. F., Johnson, S. T., Stone, A. E., Chang, E., \& Hoffman, M. A. (2020). A biomechanical comparison of dominant and non-dominant limbs during a side-step cutting task. Sports Biomechanics, 19(2), 271-279.

Pollard, C. D., Sigward, S. M., \& Powers, C. M. (2007). Gender differences in hip joint kinematics and kinetics during side-step cutting maneuver. Clinical Journal of

563 Schreurs, M. J., Benjaminse, A., \& Lemmink, K. A. (2017). Sharper angle, higher risk? The effect of cutting angle on knee mechanics in invasion sport athletes. Journal of Biomechanics, 63, 144-150. 
566 Sigward, S. M., Cesar, G. M., \& Havens, K. L. (2015). Predictors of frontal plane knee moments during side-step cutting to 45 and 110 degrees in men and women: Implications for anterior cruciate ligament injury. Clinical Journal of Sport Medicine, 25, 529-534. https://doi.org/10.1097/jsm.0000000000000155

570 Sigward, S. M., Pollard, C. D., \& Havens, K. L. (2012). The influence of sex and maturation on knee mechanics during side-step cutting. Medicine and Science in Sports and Exercise, 44, 1497-1503. https://doi.org/10.1249/MSS.0b013e31824e8813

Stuelcken, M. C., Mellifont, D. B., Gorman, A. D., \& Sayers, M. G. L. (2016). Mechanisms of anterior cruciate ligament injuries in elite women's netball: A systematic video analysis. Journal of Sports Sciences, 34(16), 1516-1522.

Thomas, C., Dos'Santos, T., Kyriakidou, I., Cuthbert, M., Fields, C., \& Jones, P. A. (2017). An investigation into the effect of limb preference on knee mechanics and braking strategy during pivoting in female soccer players: An exploratory study. Presented at the The 8th Annual Strength and Conditioning Student Conference, Middlesex University, London.

582 Winter, D. A. (2009). Biomechanics and motor control of human movement.

583 Yu, B., Lin, C. F., \& Garrett, W. E. (2006). Lower extremity biomechanics during the 584 landing of a stop-jump task. Clinical Biomechanics, 21, 297-305. https://doi.org/10.1016/j.clinbiomech.2005.11.003 
